\title{
Contribution of individual excitatory synapses on dendritic spines to electrical signalling
}

(Contribution of spine synapse to electrical signalling)

\section{Ju-Yun Weng, Cesar Ceballos, and Dejan Zecevic}

\section{Affiliations:}

Department of Cellular and Molecular Physiology, Yale University School of Medicine, New Haven, CT 06510

*Correspondence to:

Dejan Zecevic, Yale University Sch. Med., Dept. C/M Physiology, 333 Cedar St., New Haven, CT 06520.

Phone: (203)785-4486

email: dejan.zecevic@yale.edu

Number of pages: 40

Number of figures: 6

Number of words: Abstract: 211; Introduction: 629; Discussion: 1388

Conflict of interest: none

Acknowledgement: We are grateful to Lawrence B Cohen, (Yale University) for continuous support and expert advice and to Leslie M. Loew (Centre for Cell Analysis and Modelling, UConn Health Centre) for kindly providing voltage-sensitive dyes. This work was supported by National Institute of Health MH106906.

Ju-Yun Weng current affiliation: Brain Research Center, National Tsing Hua University, Hsinchu, 30013, Taiwan, Telephone: 886-3-5742478, Email: wwjd@ gapp.nthu.edu.tw Cesar Ceballos current affiliation: Vollum Institute, Oregon Health and Science University, Portland, OR, USA, Telephone: (203)910-2508, Email: ceballoc@ ohsu.edu 


\begin{abstract}
Even though dendritic spines are widely considered the elementary computational units of the brain, current understanding of the exact contribution of individual synapses on spines to the electrical signalling in neurons is incomplete. We combined the techniques of whole cell recording and voltage imaging to monitor directly unitary excitatory postsynaptic potentials evoked by two-photon glutamate uncaging (uEPSPs) on individual dendritic spines. We analysed the initiation, temporal summation, and propagation of uEPSPs from the spine head to the parent dendrite and to the cell body in neocortical pyramidal neurons. Our measurements show no significant attenuation of uEPSPs across the spine neck in different types of pyramidal neurons. This result implies that spine synapses are not electrically isolated from parent dendrites and that spines do not serve a meaningful electrical role. Using the same techniques, we next characterized temporal summation of uEPSPs induced at individual spine synapses by repetitive glutamate uncaging mimicking burst activity of presynaptic neurons. We found that pharmacological block of desensitization of AMPA receptors increased the amplitude of cumulative uEPSP response in the spines head and, consequently, in parent dendrite and soma. Thus, responses to high frequency repetitive quantal EPSPs are strictly limited in amplitude and waveform by AMPA receptor desensitization at the site of origin thereby preventing synaptic saturation.
\end{abstract}

\title{
Significance Statement
}

We used an electrochromic voltage-sensitive dye, which acts as a transmembrane optical voltmeter, to define the electrical role of dendritic spines, small membrane protrusions that 
receive most of the excitatory synaptic inputs in the brain. The data argue that spine synapses of investigated principal neurons are not electrically isolated from the parent dendrites. We also found that AMPA receptor desensitization restricts the amplitude of temporal summation during repetitive synaptic activation. These results facilitate our understanding of how a complex assembly of receptors and ion channels in spines are utilized to generate and process electrical signals and mediate plasticity in response to quantal release of chemical transmitters caused by patterned activity in presynaptic axons.

\section{Introduction}

The objective of this study is to characterise the contribution of single spine synapses to the electrical signalling of individual neurons and define the electrical role of dendritic spines. Several studies (see below) hypothesized that dendritic spines serve special functions in electrical signalling because synapses on spine heads are electrically isolated from the parent dendrite by the slender spine neck. Other studies (see below) provide evidence that spine synapses are not electrically isolated and that spines have no electrical role. At present, neither one of these opposing postulates is universally accepted.

The hypothetical electrical role of spines implies high electrical resistance of the $\sim 1 \mu \mathrm{m}$ long spine neck cable $\left(\mathrm{R}_{\text {neck }}\right)$ relative to the input impedance of the $\sim 60-1000 \mu \mathrm{m}$ parent dendritic cable $\left(Z_{\text {dendrite }}\right)$. A high value of $R_{\text {neck }}$ would also imply the functional significance of highly variable morphology of individual spine necks, making practically every spine functionally different.

One group of studies, supporting electrical role of spines, postulate high $\mathrm{R}_{\text {neck }}$ partially or completely on theoretical grounds. The results of numerical simulations in neurophysiology, as a 
rule, depend on a number of uncertain approximations/assumptions (Koch et al., 1983;

Bloodgood and Sabatini, 2005; Grunditz et al., 2008; Bloodgood et al., 2009; Xu et al., 2012;

Gulledge et al., 2012; Araya et al., 2014; Tønnesen et al., 2014; Acker et al., 2016; Cartailler et al., 2018; Lagache et al., 2019). Another group of studies postulate high $\mathrm{R}_{\text {neck }}$ based on indirect measurements that are difficult to interpret unambiguously (Bloodgood and Sabatini, 2005; Araya et al., 2006a, 2006b, 2014; Grunditz et al., 2008; Bloodgood et al., 2009; Harnett et al., 2012; Tønnesen et al., 2014; Bywalez et al., 2015; Acker et al., 2016; Hage et al., 2016; Beaulieu-Laroche and Harnett, 2018). A third group of studies postulate high $\mathrm{R}_{\text {neck }}$ based on the results from electrical and optical recordings that require extensive corrections (Jayant et al., 2016; Kwon et al., 2017; Cartailler et al., 2018; Cornejo et al., 2022).

In contrast, a different set of reports provides indirect evidence that, in great majority of spines $(>80 \%)$, the neck resistance is too small relative to the input impedance of the dendrite to affect synaptic signals. Some of these studies are based on theoretical considerations and numerical simulations (Rall and Rinzel, 1973; Rall, 1974; Koch and Zador, 1993) while others are based on experimental measurements of diffusional resistance of the spine neck (Svoboda et al., 1996; Takasaki and Sabatini, 2014; Tønnesen et al., 2014; Miyazaki and Ross, 2017). The interpretation of modelling results and indirect data from these studies, by necessity, rest on assumptions. In agreement with these findings, we recently provided the first direct evidence for low electrical resistance of the spine neck using voltage-sensitive dye recordings characterized by adequate sensitivity and spatiotemporal resolution (Popovic et al., 2015b). This technique uses an organic voltage-sensitive dye that acts as a transmembrane voltmeter with a linear scale in the entire physiological range of neuronal membrane potential signals. The traces showing fluorescent light intensity changes from the voltage-sensitive probe track the membrane potential 
transients exactly, in a manner similar to traces made on an oscilloscope screen by amplified transmembrane voltage transients recorded by an intracellular electrode. This method allows monitoring voltage transients simultaneously at both ends of the spine neck, i.e. in the spines head and in the parent dendrite at the base of the spine. Under our recording conditions, the conclusions from these results do not depend on any assumptions. In this report, we confirm the absence of electrical role of spine necks in L5 pyramidal neurons and document that same conclusions are valid for other classes of principal nerve cells in the mammalian cortex. Using the same technique, we then investigated the contribution of repetitive activation of individual excitatory synapses on spines to electrical signalling in neurons.

\section{Materials and Methods}

Experimental design and statistical analysis. We used high sensitivity voltage imaging with an organic electrochromic dye to analyze the electrical role of dendritic spines in selectively labeled L2/3, L5 and L6 pyramidal neurons in rat cortical slices. Statistical analyses were performed using GraphPad Prism 9.3.1. All values are reported as mean \pm SEM.

Slices, patch-clamp recording and intracellular application of dyes. All surgical and experimental procedures were performed in accordance with Public Health Service Policy on Humane Care and Use of Laboratory Animals and approved by Yale University Institutional Animal Care and Use Committee. Experiments were carried out on somatosensory cortex slices from 18-30-day old rats of either sex. The animals were decapitated following deep isoflurane anaesthesia, the brain was quickly removed, and $300 \mu \mathrm{m}$ thick coronal cortical slices were cut in ice-cold solution using a custom-made rotary slicer with circular blade (Specialty Blades Inc., Staunton, VA). Slices were incubated at $34^{0} \mathrm{C}$ for $\sim 30$ minutes and then maintained at room 
temperature $\left(23-25^{0} \mathrm{C}\right)$. The standard extracellular solution used during recording contained (in $\mathrm{mM}): 125 \mathrm{NaCl}, 25 \mathrm{NaHCO}_{3}, 20$ glucose, $2.5 \mathrm{KCl}, 1.25 \mathrm{NaH}_{2} \mathrm{PO}_{4}, 2 \mathrm{CaCl}_{2}$ and $1 \mathrm{MgCl}_{2}, \mathrm{pH} 7.4$ when bubbled with a $5 \% \mathrm{CO}_{2}$ gas mixture balanced with $95 \% \mathrm{O}_{2}$. Somatic whole-cell recordings in current clamp or voltage-clamp mode were made with 4-6 M $\Omega$ patch pipettes using a Multiclamp 700B amplifier (Axon Instruments Inc., Union City, CA). Voltage-clamp recordings were made with series resistance compensation set at $70 \%$. The pipette solution contained (in mM): 120 K-gluconate, $3 \mathrm{KCl}, 7 \mathrm{NaCl}, 4 \mathrm{Mg}$-ATP, $0.3 \mathrm{Na}-\mathrm{GTP}, 20$ HEPES and 14 Trisphosphocreatine ( $\mathrm{pH} 7.3$, adjusted with $\mathrm{KOH}$ ) and $0.8 \mathrm{mM}$ of the voltage-sensitive dye JPW3028 (Antić and Zecević, 1995). The pharmacological agents were obtained from Tocris. The somatic whole cell recording data were not corrected for liquid junction potential. We selected pyramidal cells with intact dendrites in one plane of focus close to the surface of the slice (to minimize light scattering) using infrared differential-interference contrast (DIC) video-microscopy. The recordings were carried out from spines on superficial basal dendrites at different distances from the soma (range $60-230 \mu \mathrm{m}$ ). Individual pyramidal neurons were labelled with the membrane impermeant voltage sensitive dye by allowing free diffusion of the probe from the somatic patch pipette in the whole-cell configuration. We used the most successful voltage probe for intracellular application, JPW3028, a close analogue of JPW1114 (Zecević, 1996) with similar voltage sensitivity available from Invitrogen as D6923. Glass pipettes were first filled from the tip with dye-free solution by applying negative pressure for about 15 seconds and then backfilled with the solution containing the indicator dye $(0.8 \mathrm{mM})$. Intracellular staining was accomplished in 15-60 minutes, depending on electrode access resistance. After enough dye diffused into the cell body, as determined by measuring resting fluorescence intensity from the soma, the patch-electrode was detached from the neuron by forming an outside-out patch. The 
staining level was determined empirically as a compromise that attains an adequate level of fluorescence without causing damage by prolonged dialysis from the patch pipette. The preparation was typically incubated for an additional 1.5 - 2 hours at room temperature to allow the voltage-sensitive dye to spread and equilibrate in the dendritic arbour. To obtain electrical recordings, the cell body was re-patched using an electrode filled with dye-free intracellular solution before making optical measurements at $34 \mathrm{C}^{\circ}$. APs and steady-state hyperpolarizing signals were evoked by transmembrane current pulses delivered via the recording electrode attached to the soma in whole cell configuration.

Optical recording. The recording setup was built around a stationary upright microscope (Olympus BX51; Olympus Inc., USA) equipped with a high spatial resolution CCD camera for infrared DIC video microscopy and a high-speed data acquisition camera used for voltage imaging. This camera (NeuroCCD-SM, RedShirtImaging LLC, Decatur, GA, USA) is characterized with relatively low spatial resolution ( $80 \times 80$ pixels), exceptionally low read noise, and a full frame rate of $2 \mathrm{kHz}$. The frame rate can be increased to $5 \mathrm{kHz}$ by reading out the central subsection of the camera chip of $24 \times 80$ pixels. The $5 \mathrm{kHz}$ recording mode was used in all experiments so that signals can be accurately reconstructed for calibration and comparison. The brain slice was placed on the stage of the microscope and the fluorescent image of the stained neuron projected by a water dipping objective onto the CCD positioned in the primary image plane. For optical recordings from individual spines, we used a 100X/1.0 NA Olympus objective. This objective was selected as a compromise between imaging area, spatial resolution and signal-to-noise ratio $(\mathrm{S} / \mathrm{N})$. Optical recording was carried out in the wide-field epi-fluorescence microscopy mode. A frequencydoubled $500 \mathrm{~mW}$ diode-pumped $\mathrm{Nd}$ : YVO4 continuous wave $(\mathrm{CW})$ laser emitting at $532 \mathrm{~nm}$ (MLL532, Changchun New Industries Optoelectronics Tech. Co., Ltd., Changchun, China) was 
the source of excitation light. The laser beam was directed to a light guide coupled to the microscope via a single-port epifluorescence condenser (TILL Photonics GmbH, Gräfelfing, Germany) designed to provide approximately uniform illumination of the object plane. The laser was used as a light source in place of a conventional Xenon arc-lamp to increase the sensitivity of Vm-imaging by: (1) providing a monochromatic excitation light at the red edge of the absorption spectrum to maximize Vm sensitivity of the dye (Loew, 1982; Kuhn et al., 2004; Holthoff et al., 2010; Popovic et al., 2015b) and (2) increasing the intensity of the excitation light beyond the level that can be achieved by an arc-lamp. Excitation light was reflected to the preparation by a dichroic mirror with a central wavelength of $560 \mathrm{~nm}$ and the fluorescence light was passed through a band pass emission filter (FF01-720; 720 nm blocking edge BrightLine, Semrock). For voltage imaging, laser light was gated by a high-speed shutter (Uniblitz LS6, driver D880C). Acquisition and analysis of data were carried out using NeuroPlex software (RedShirtImaging). In this configuration a CCD frame ( 80 x 80 pixels) corresponded to a field of $18 \times 18 \mu \mathrm{m}$ in the object plane with each individual pixel receiving light from an area of $\sim 0.23 \times 0.23 \mu \mathrm{m}$ in the focal plane.

Computer-generated holography for two-photon uncaging of glutamate. The voltage imaging setup was integrated with an ultra-fast pulsed titanium-sapphire laser tuned to $720 \mathrm{~nm}$ for 2-photon glutamate uncaging (Chameleon Ultra, Coherent Inc.). The light intensity of the laser and the duration of the uncaging pulse were controlled by a Pockells cell (Model 350-80, Conoptics Inc.). The two-photon uncaging pattern was generated using a commercial module for holographic illumination (Phasor-3i Intelligent Imaging Innovation, Inc. Denver, CO USA), modulating 720 nm laser source controlled by SlideBook software (3i Intelligent Imaging Innovation). We used multipoint patterns acquired on the NeuroCCD camera to calibrate the exact positioning of the holographic spots. By introducing a correcting stretch, translation, and rotation transformation to 
the input patterns that was provided to the Phasor algorithm, we could achieve submicron precision in spot positioning. We estimated the effective excitation beam size based on the measured experimental profile to be $\sim 0.6 \mu \mathrm{m}$ in diameter. The exact focal volume of 2-photon excitation (Matsuzaki et al., 2001) was not determined but the spatial resolution of glutamate uncaging was sufficient to activate one spine synapse in isolation as it has been repeatedly documented (Matsuzaki et al., 2001; Smith et al., 2003; Carter and Sabatini, 2004; Sobczyk et al., 2005; Tazerart et al., 2020) and shown here in Figure 2. The wide field illumination was used to obtain an image of dye-loaded dendrites and identify structures of interest for glutamate uncaging and voltage imaging. DNI-glutamate TFA provided by Femtonics KFT (Budapest, Hungary), which has $\sim 7$ times higher 2-photon uncaging efficiency (Chiovini et al., 2014) than the commonly used MNI caged compound, was bath applied at concentration of $4 \mathrm{mM}$. The illumination spots were placed at a distance of $\sim 0.5 \mu \mathrm{m}$ from individual spine heads. The actual spatial relationship between the uncaging spots and spine heads was uncertain at the sub-micrometer spatial scale because of light scattering in the brain tissue. The uncaging light pulse was adjusted in duration (from 0.2-0.5 ms) and intensity (from 10-20 $\mathrm{mW}$ under the objective) to produce a response similar to a unitary EPSP in the soma (0.2-0.8 mV) (Magee and Cook, 2000; Nevian et al., 2007; Bloodgood et al., 2009; Enoki et al., 2009).

At the end of each experiment, a detailed morphological reconstruction of dye loaded neurons was carried out on a stationary upright microscope (AxioExaminer D1 with zoom tube $(0.5-4 x)$, Carl Zeiss Microscopy LLC) equipped with a high spatial resolution CCD camera (1392x1024 pixels; Pixelfly-qe, PCO Imaging, Kelheim, Germany) mounted on a spinning-disc confocal scanner (Yokogawa CSU-10). At the end of every experiment, this system was used to collect z-stacks of confocal images for detailed morphological reconstruction of basal dendrites 
and spines. Morphological reconstruction was used to determine the distance of the recording site from the soma and calibrate optical signal in terms of membrane potential using the known bAP amplitude at the corresponding distances (see below).

Data analysis. Subthreshold uncaging evoked excitatory postsynaptic potential signals (uEPSP) were recorded typically for $60 \mathrm{~ms}$ at a frame rate of $5 \mathrm{kHz}$ at near physiological temperature of 32-34 $\mathrm{C}^{0}$. Analysis and display of data were carried out using the NeuroPlex program (RedShirtImaging) written in IDL (Exelis Visual Information Solutions, Boulder, CO) and custom Visual Basic routines. Background fluorescence can be a significant determinant of $\Delta \mathrm{F} / \mathrm{F}$ signal size. Raw data were first corrected for this effect by subtracting the average background fluorescence intensity determined from an unstained area on the slice. Subsequently, signal alignment software was used to correct for temporal jitter in AP initiation as well as for possible small movements of the preparation during averaging (Popovic et al., 2015b). In the temporal domain, AP signals were aligned by cross correlation of the electrically recorded APs in each trial to the reference signal acquired at the start of averaging. In the spatial domain, camera images were aligned in two dimensions offline by image cross-correlation to compensate for possible small lateral movements of the preparation (Popovic et al., 2015a). Correct focus of the image in the z-dimension was verified before each individual trial; small adjustments were often necessary. The spatially and temporally aligned signals were averaged and slow changes in light intensity due to bleaching of the dye were corrected by dividing the data by an appropriate dual exponential function derived from the recording trials with no stimulation (Grinvald et al., 1982). The waveform of the AP signal was reconstructed from a set of data points using Cubic Spline Interpolation, a piecewise continuous curve passing through each data point (Popovic et al., 2011). Subthreshold optical signals were calibrated on an absolute scale (in 
$\mathrm{mV}$ ) by normalizing to an optical signal from a backpropagating action potential (bAP) which has a known declining amplitude along basal dendrites, as previously determined by patchpipette recordings (Nevian et al., 2007; Palmer and Stuart, 2009). These methods of calibration produces the same results as normalizing signals to optical recordings corresponding to long hyperpolarizing pulses delivered to the soma which attenuate relatively little as they propagate along dendrites (Stuart and Spruston, 1998; Nevian et al., 2007; Palmer and Stuart, 2009; Holthoff et al., 2010).

\section{Results}

\section{Spine synapses are not electrically isolated from dendrites}

The goal of the first series of experiments was to extend our previous conclusions that spines are not isolated electrical compartments (Popovic et al., 2015b) to other classes of cortical pyramidal neurons. The experiments were carried out on basal dendrites of L2/3, L5, and L6 cortical pyramidal neurons. The only direct way to answer the question of the electrical isolation of spine synapses is to simultaneously record EPSP signals from the spine head and from the parent dendrite at the base of the spine following strictly selective quantal activation of a single synapse. Under these conditions, the evoked EPSP signal in the spine head represents a voltage drop caused by the synaptic current flow across $\mathrm{R}_{\text {neck }}$ and $\mathrm{Z}_{\text {dendrite }}$ connected in series according to the expression:

$$
\mathrm{EPSP}_{\text {spine }}=\mathrm{I}_{\text {syn }}\left(\mathrm{R}_{\text {neck }}+\mathrm{Z}_{\text {dend }}\right)
$$

The EPSP signal in the parent dendrite at the base of the spine represents a voltage drop caused by the same synaptic current flow across $\mathrm{Z}_{\text {dendrite }}$ alone according to the expression: 


$$
\text { EPSP }_{\text {dend }}=I_{\text {syn }} Z_{\text {dend }}
$$

(2) (Koch and Zador, 1993).

According to these two expressions, the experimental approach to the question of the electrical role of spines is conceptually trivial, even though it is technically demanding to a degree that prevented these measurements for several decades. If one can simultaneously record EPSP spine $_{\text {in }}$ and $\mathrm{EPSP}_{\text {dendrite, }}$, one can anticipate two different categories of results. One possible result is that the EPSP amplitude is significantly larger in the spine head than in the parent dendrite. This result would imply a relatively large $R_{\text {neck}}$, comparable to $Z_{\text {dendrite }}$ at a given dendritic location.

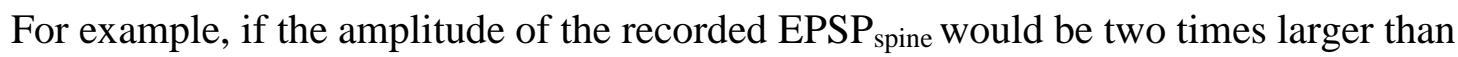
$\mathrm{EPSP}_{\text {dendrite, }}$ that would mean that $\mathrm{R}_{\text {neck }}$ is equal to $\mathrm{Z}_{\text {dendrite. }}$. The other possible result is that

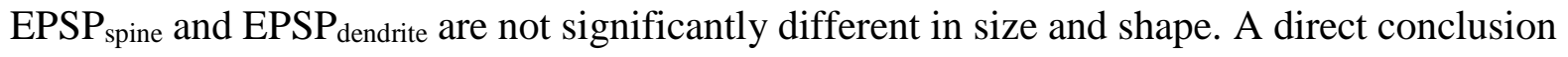
from this type of a result would be that $R_{\text {neck }}$ must be negligible compared to $Z_{\text {dendrite. }}$.

We used 2-photon uncaging of glutamate and high sensitivity organic voltage-sensitive dye recordings to monitor simultaneously uncaging evoked quantal EPSP signals (uEPSP) from individual dendritic spine heads and parent dendrites at the base of the spine. Optical recordings carried out from pyramidal neurons at a frame rate of $5 \mathrm{kHz}$ (using a central subsection of 24x80 pixels of the NeuroCCD-SM; Methods) improved the accuracy of our earlier results obtained at the sampling rate of $2 \mathrm{kHz}$ (Popovic et al., 2015b). The results (Fig. 1) documented that the method has adequate sensitivity at the required spatiotemporal resolution to simultaneously record and faithfully reconstruct individual EPSPs and APs signals from spine heads and parent dendrites at near physiological temperature. It is noteworthy that, presently, there is no other recording methodology that can accomplish this goal. Fig. 1A illustrates optical recordings of the uEPSP signal evoked by 2-photon uncaging of glutamate adjusted in light intensity and duration (Methods) to mimic quantal glutamate release resulting from the arrival of one AP at the 

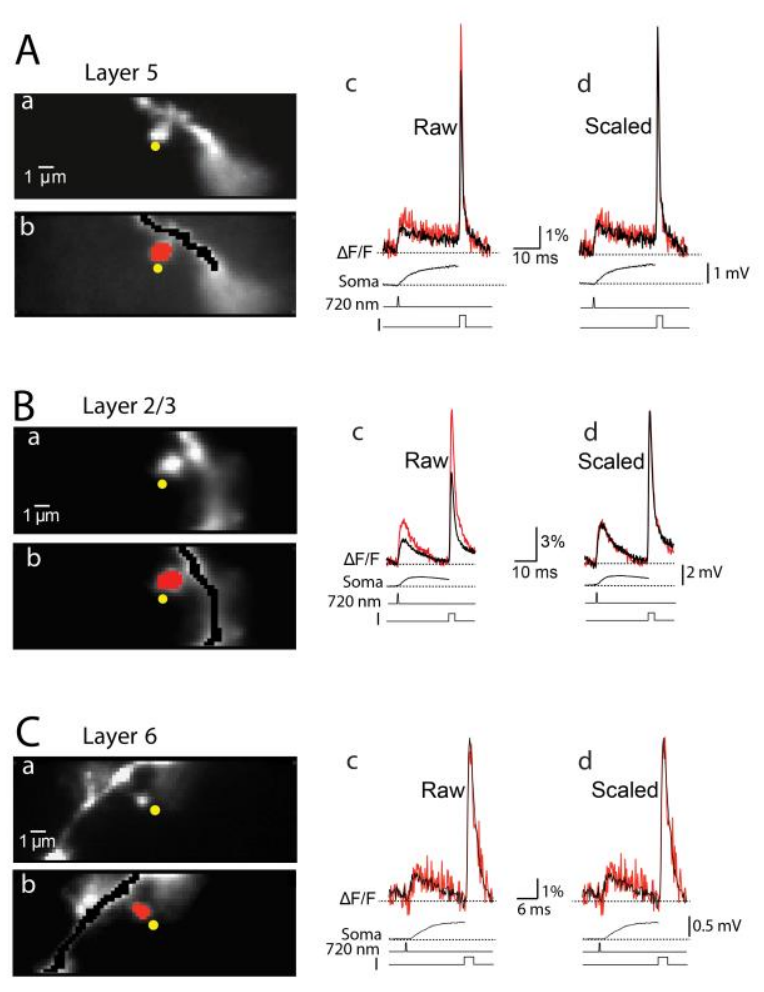
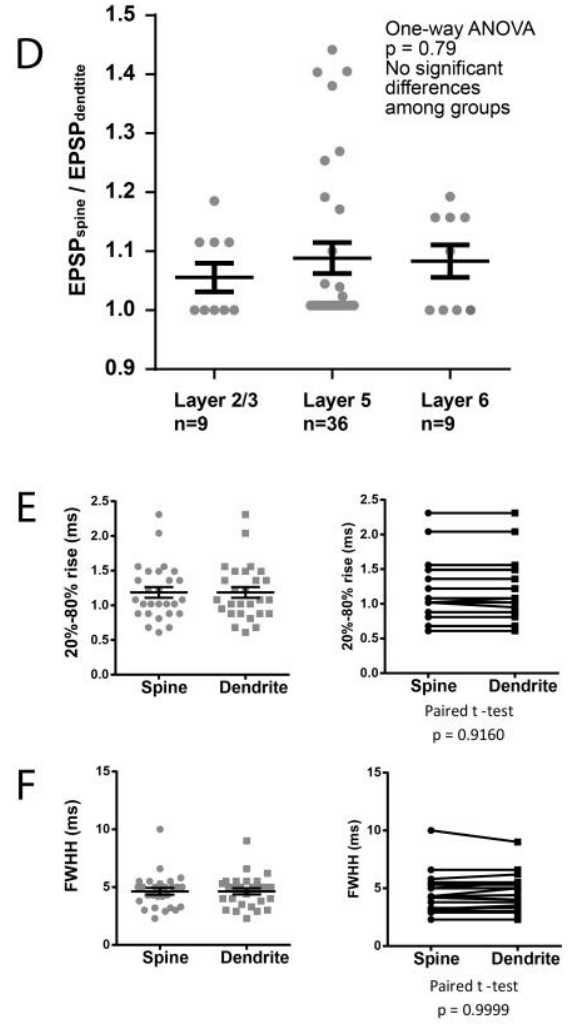

Figure 1. Optical recordings of EPSP and AP signals from individual spines and parent dendrites from cortical L5, L2/3, and L6 pyramidal neurons. A, L5 pyramidal neuron. (a) Fluorescence image of a spine in recording position obtained with CCD for voltage imaging. Yellow dots indicate the position of the $720 \mathrm{~nm}$ light spot $600 \mathrm{~nm}$ in diameter, used for 2-photon glutamate uncaging. (b) Selection of pixels used for the spatial average of optical signals from the spine head (red) and parent dendrite (black). (c) Evoked uEPSP and AP signal from spine head and parent dendrite superimposed. Bottom black traces: Top: electrode recording of somatic uEPSP. Middle: the uncaging command pulse. Bottom: transmembrane current pulse delivered by a somatic patch electrode. (d) Superimposed signals from the spine head and parent dendrite corrected for recording sensitivity difference by normalizing to the bAP optical signal. $\boldsymbol{B}$ and $\boldsymbol{C}$, Similar recordings from L2/3 and L6 neurons, respectively. D, Scatter plot of individual values of the ratio (uEPSPspine/uEPSPdendrite) for three classes of cortical pyramidal neurons. Vertical lines show mean \pm SEM). E, F, Scatter plot of individual values of uEPSP 20-80\% rise time (E) and full width at half height $(F W H H)(F)$. Vertical lines show mean \pm SEM. 
presynaptic bouton. Immediately following the uEPSP, within the same $60 \mathrm{~ms}$ recording period, we evoked a bAP by a brief depolarizing current pulse delivered from the somatic patch electrode. In all measurements, we used the bAP signals in spines and dendrites to normalize the sensitivity of optical recordings from different locations (scaled signals). This normalization is based on prior knowledge that bAP has the exact same size and shape in the spine and the parent dendrite (Palmer and Stuart, 2009; Popovic et al., 2014). The experiment in Fig. 1A shows clearly that there was very little or no detectable difference in both amplitude and kinetics

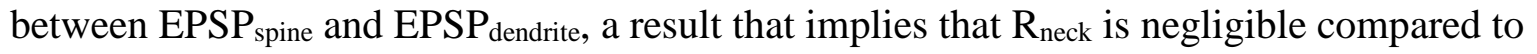
$Z_{\text {dendrite. }}$ The same result was obtained from five L5 pyramidal neuron measurements from 5/5/5 spines/neurons/animals. When combined with our previous data from L5 pyramidal neurons

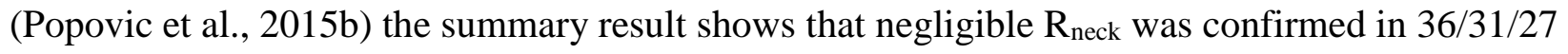
spines/neurons/animals. The average ratio EPSP $_{\text {spine/ }}$ EPSP $_{\text {dend }}$ Was $1.08 \pm 0.03(n=36)$. We next examined, in the same way, two additional classes of cortical neurons. As in L5 neurons, we found very little or no detectable difference in shape and size between EPSP $_{\text {spine }}$ and EPSP $_{\text {dendrite }}$ in measurements from spines from basal dendrites of L2/3 and L6 pyramidal neurons. The average ratio $\mathrm{EPSP}_{\text {spine }} / \mathrm{EPSP}_{\text {dend was }} 1.05 \pm 0.02(\mathrm{n}=9)$ for $\mathrm{L} 2 / 3$ and $1.08 \pm 0.03(\mathrm{n}=9)$ for L6 neurons. The scatter plots showing the distribution of individual values for the ratio of amplitudes and for the kinetics (rise time and FWHH) for EPSP spine $_{\text {and }}$ EPSP dend $_{\text {are shown in }}$ Fig. 1D-F. Taken together, the results from basal dendrites of three different classes of principal cortical neurons provide direct evidence that $R_{\text {neck }}$ is insignificant relative to $Z_{\text {dendrite }}$ and that synapses on spine heads are not electrically isolated from the parent dendrite to a degree that would have functional meaning. These findings, when supplemented by the necessary control experiments described below, do not depend on any assumptions. We investigated three possible sources of errors, which might influence the accuracy of our measurements and the validity of our conclusions: (1) insufficient sensitivity of voltage imaging; (2) contribution of extrasynaptic receptors; (3) insufficient spatial resolution of voltage imaging. 


\section{Sensitivity of optical recording}

The accuracy of our measurements depends directly on the sensitivity of optical recording at the spatiotemporal resolution required for the accurate reconstruction of electrical signals from optical data. Figure 1 indicates that our recordings at the spatial scale of individual spines and at the frame rate of $5 \mathrm{kHz}$ can resolve both bAP signals and EPSP signals with the signal-to-noise ratio that varied from 3 to 20 in different experiments. We have documented previously that a sampling rate of $5 \mathrm{kHz}$ (but not 1 or $2 \mathrm{kHz}$ ) was adequate for accurate reconstruction of the bAP in both dendrites and spines (see Fig. 4 in Popovic et al. 2014). This is a critical issue because an accurate AP signal is mandatory for normalizing sensitivity of optical recording from different locations. It follows that uEPSP signal reconstruction was also accurate because uEPSPs have slower dynamics then bAP signals. Thus, it is safe to conclude that the comparison of uEPSP from spines and dendrites, after normalizing the recording sensitivity from different regions, was accurate.

\section{Contribution of extrasynaptic receptors}

The validity of our results depends on strict spatial selectivity of 2-photon uncaging of glutamate. If 2-photon uncaging on spine head synapses also activated extrasynaptic receptors on parent dendrites to a significant degree, this effect would contribute to the similarity of responses from spines and dendrites. Both pioneering and the most recent studies firmly established single spine spatial resolution of 2-photon uncaging (Matsuzaki et al., 2001; Smith et al., 2003; Tazerart et al., 2020). Several laboratories investigated and clearly documented that non-specific activation of glutamate receptors on parent dendrites is so small that it can be safely neglected (Matsuzaki et al., 2001; Sobczyk et al., 2005; Popovic et al., 2015b). 
A
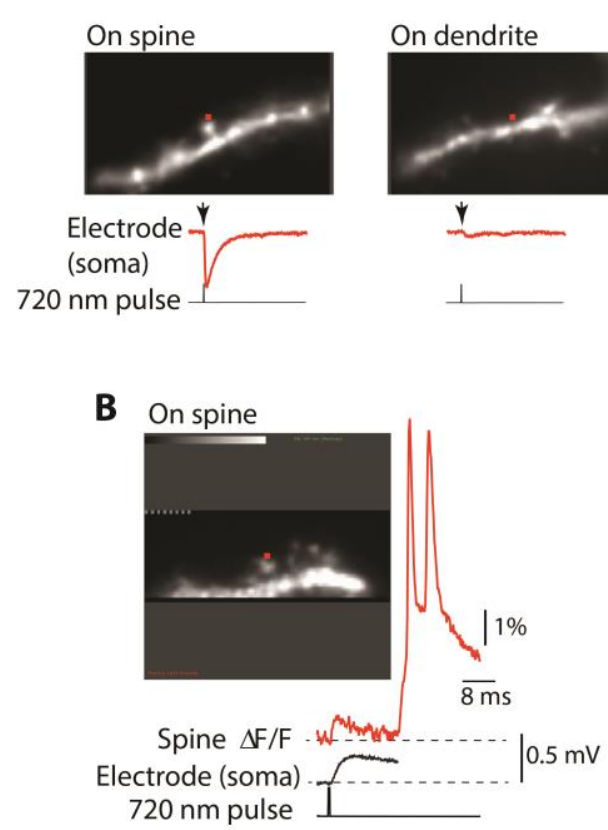

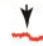

L5 Pyramidal neuron
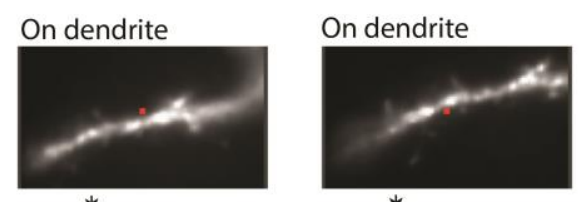

$\underline{y}$

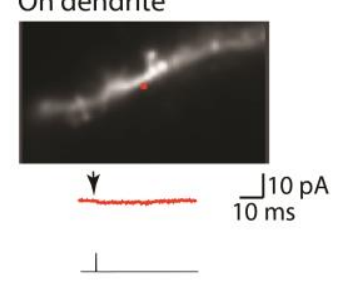

C Off spine

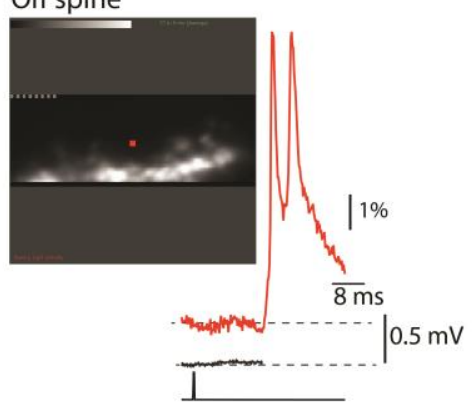

D

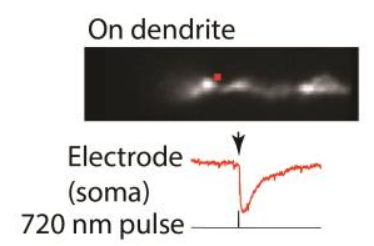

L1 Aspiny interneuron

On dendrite

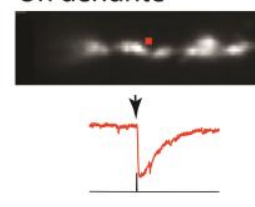

On dendrite

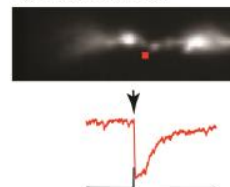

On dendrite

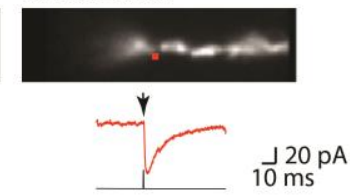

Figure 2. Selective activation of individual synapses. A, Uncaging glutamate directly on smooth, aspiny regions of basal dendrites of L5 pyramidal neurons (On dendrite) evoked currents (red traces recorded under voltage-clamp) that were either not detectable or represented a very small fraction of the current evoked by activating synapses on neighboring spines (On spine). Red dots indicate the position of the $720 \mathrm{~nm}$ light spot used for 2-photon glutamate uncaging. $\boldsymbol{B}$, Uncaging glutamate on an individual spine evokes clear uEPSP as revealed by voltage imaging (red trace) and electrical recording from the soma (black trace). $\boldsymbol{C}$, Uncaging glutamate at a distance from aspiny dendritic regions equivalent to the distance of the spine head failed to produce measurable uEPSP responses in both optical and electrical recordings. D, Uncaging glutamate on aspiny dendrites on L1 interneurons evoked fast and clear response at all locations. 
We confirmed this conclusion in voltage-clamp experiments (Fig. 2A) showing that uncaging glutamate directly on aspiny regions of basal dendrites of pyramidal neurons evoked currents that were either not detectable or represented a very small fraction $(4 \% \pm 0.9 \% ; n=34)$ of the current evoked by activating synapses on neighboring spines. Moreover, uncaging glutamate at a distance from aspiny dendritic regions of pyramidal neurons equal to the distance of the spine head failed to produce measurable uEPSP responses (Fig. 2B, C). This result was confirmed in 9 experiments. In contrast to these results on pyramidal neurons, and as a positive control, we found that uncaging glutamate directly onto aspiny dendrites on L1 interneurons evoked fast and clear response at all locations tested in $n=18$ interneurons (Fig. 2D). This result is in line with previous reports showing that AMPA glutamate receptors are largely absent from extrasynaptic regions on basal dendrites of cortical pyramidal neurons (Sobczyk et al., 2005; Popovic et al., 2015b) while they are widely distributed along densely innervated smooth dendrites of aspiny interneurons (Gulyás et al., 1999; Sancho and Bloodgood, 2018).

\section{Light scattering and spatial resolution}

Light scattering in the brain tissue limits the spatial resolution of voltage imaging in wide-field microscopy mode. Here, because the uEPSP in the dendrite is secondary to the uEPSP in the spine and, hence, must be always smaller, the relevant question is whether fluorescent light from spine heads contaminated optical signals from parent dendrite, making it artificially bigger. This type of crosstalk, if significant, would tend to make the signals from spines and dendrites similar. We have previously established that the extent of this contamination in the superficial layers of the slice, less than $30 \mu \mathrm{m}$ deep, was very small and often not detectable ( 3\%; see Figs. 3 in 
Popovic et al. 2014; 2015b). The crosstalk in the opposite direction (from parent dendrite to the spine head) was also shown previously to be small in superficial layers of the slice $(<10 \%$; see Figs. 3 in Popovic et al. 2014; 2015b) and without significant effect on our conclusions.

Taken together, our control measurements show that optical recordings of quantal uEPSP signals and bAP signals are accurate. It is important to emphasize again that direct comparison showing similar size and shape of uEPSP signals from spine heads and parent dendrites due to $\mathrm{R}_{\text {neck }}<<\mathrm{Z}_{\text {dendrite }}$ does not depend on any assumptions. The ratio (uEPSPspine/uEPSPdendrite) does not depend on the position and distance of the spine from the soma, does not depend on absolute values of $\mathrm{R}_{\text {neck }}$ and $\mathrm{Z}_{\text {dendrite }}$ and does not require calibration of optical signals in terms of membrane potential. Thus, possible errors due to unavoidable inaccuracies in calibrating optical signals in terms of membrane potential (Popovic et al., 2015b; Acker et al., 2016; Kwon et al., 2017) can safely be ruled out. The data argue that uEPSPs are not attenuated significantly as they propagate from synapses on spine heads to the parent dendrite. This result implies that, in strictly electrical terms, synapses on cortical dendritic spines behave in the same way as synapses made directly on dendrites.

\section{Temporal summation at single spines}

Because presynaptic neurons often fire in burst (Kole, 2011), we investigated the results of temporal summation of uEPSPs following repetitive activation of single spine synapses by 2photon glutamate uncaging. These experiments mimic physiological conditions in which the natural sensory stimulus activates isolated individual spines on dendritic branches (Jia et al., 2010; Chen et al., 2011; Varga et al., 2011). Figure 3 illustrates a typical experimental approach for monitoring contribution of individual synapses. A fluorescence image of a L5 pyramidal 
neuron labeled with the voltage sensitive dye was used to identify an isolated spine close to the surface of the slice (Fig. 3A).
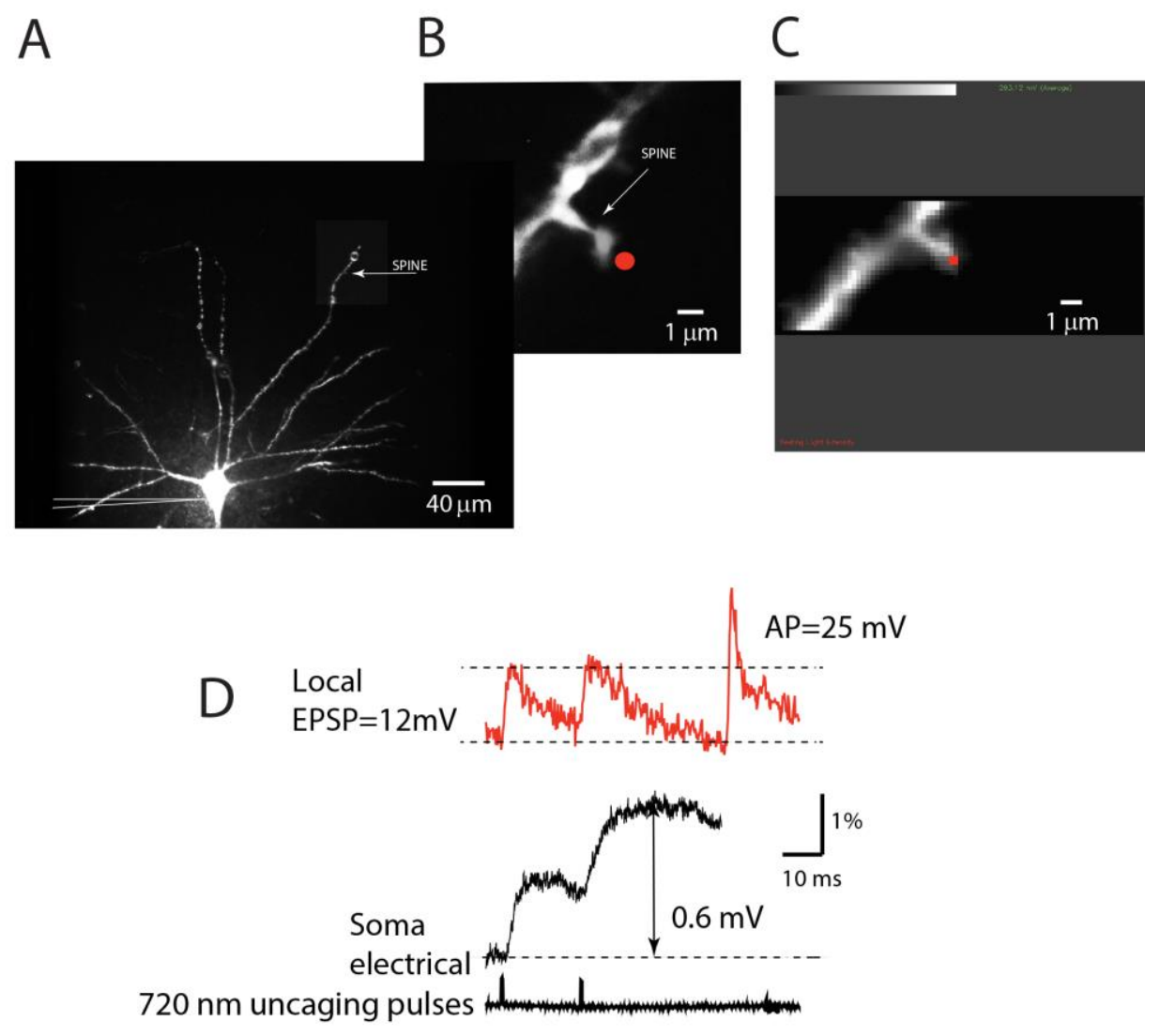

Figure 3. Contribution of individual synapses: experimental design. A, An individual neuron is labelled with a voltage sensitive dye. An isolated spine is identified in a distal region of basal dendrite under low magnification. A patch electrode (shown schematically) is attached to the soma for electrical recording and stimulation. $\boldsymbol{B}$, Fluorescent image of an isolated spine in recording position obtained at high magnification with a high-resolution $C C D . C$, Image of the same spine obtained by reading out a $24 x 80$ pixel subsection of the CCD camera for voltage imaging at $5 \mathrm{kHz}$. Red dot indicates the position of the $720 \mathrm{~nm}$ light spot used for 2-photon glutamate uncaging. $D$, Red traces: optical recordings of local uEPSP signals evoked by 2photon glutamate uncaging followed by a bAP signal evoked by depolarizing pulse delivered by the somatic patch electrode. Optical signals are spatial average of all bright pixel in the image. Black traces: somatic electrode recordings (upper) and uncaging command pulses (lower). 
Using patterned illumination based on CGH system (Methods), a $720 \mathrm{~nm}$ light spot from a pulsed laser was positioned to within $0.5 \mu \mathrm{m}$ from the edge of an individual spine for 2-photon uncaging of glutamate. A patch electrode was attached to the cell body to monitor the membrane potential and synaptic currents. The electrode also allowed us to pass depolarizing current and evoke a bAP used for normalizing recording sensitivity from different regions and for calibrating optical signals on an absolute scale (in $\mathrm{mV}$ ). This calibration is an approximation accurate to $\pm 10 \%$ because it depends on an estimate of bAP amplitude as a function of distance from the soma, which is based on published patch-electrode recordings from basal dendrites of L5 pyramidal neurons (Nevian et al., 2007). The uncaging light pulse was adjusted in duration and intensity to mimic a unitary EPSP in the soma (0.2-0.8 $\mathrm{mV}$; Methods). The uEPSP related optical signals were recorded at the site of origin as spatial average of the spine and a small region ( 15 um) of the dendrite at the base of the spine. We showed above (Fig. 1) that this entire surface is very nearly equipotential. Optical signals were recorded simultaneously with the electrode recording from the soma (Fig 3D). From this type of measurement, we established that an average quantal uEPSP recorded at the site of origin had $20-80 \%$ rise time of $1.2 \pm 0.07 \mathrm{~ms}$ and FWHH of $4.6 \pm 0.3 \mathrm{~ms}(\mathrm{n}=26)$. Scatter plots showing the distribution of individual values are shown in Fig. 1E-F. Due to rapid kinetics of EPSPs at the site of origin, there was very little or no temporal summation of signals at the synapse if the uncaging pulses were delivered with inter-pulse interval of $20 \mathrm{~ms}$ (Fig 3D). However, a clear summation was recorded at the somaaxon region because EPSP in the soma had considerably slower kinetics due to RC filtering effect in the dendritic cable (Fig. 3D). To ensure that local summation will take place, the following measurements were carried out with 5 uncaging pulses delivered at the frequency of 

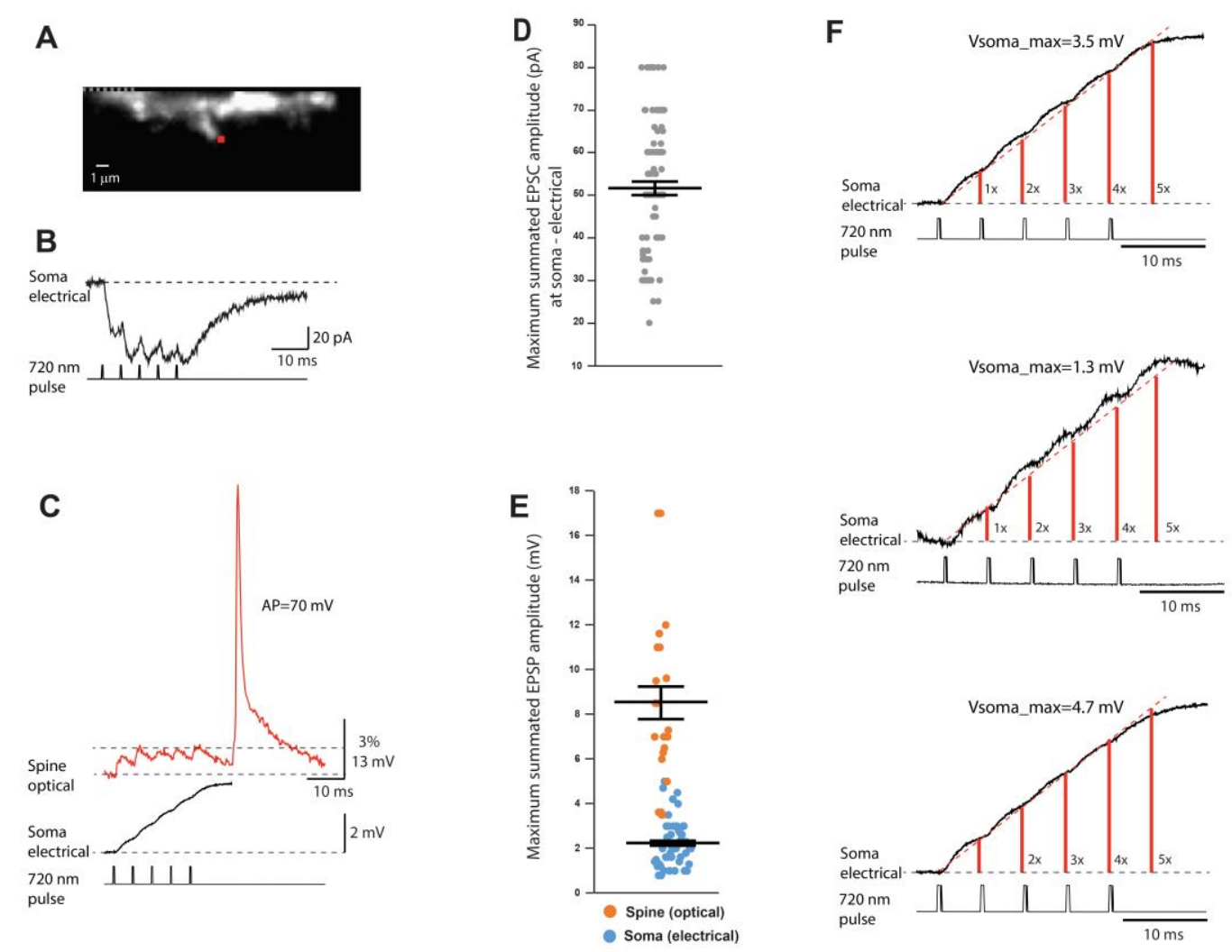

Figure 4. Summation of uEPSP signals. A, Fluorescence image of a spine in recording position. Red dot indicates the position of the $720 \mathrm{~nm}$ light spot used for 2-photon glutamate uncaging. $\boldsymbol{B}$, Upper trace: summated uEPSCs recorded with a somatic patch electrode under voltage-clamp in response to repetitive glutamate uncaging at $200 \mathrm{~Hz}$. Lower trace: command uncaging pulses. $\boldsymbol{C}$, Upper trace: Summating uEPSP related signal recorded optically from the dendritic spine and the parent dendrite section shown in (A) in response to repetitive glutamate uncaging at $200 \mathrm{~Hz}$. Middle trace: Summating uEPSP signal recorded with a somatic patch electrode simultaneously with optical recording of local signals. Bottom trace: Uncaging command pulses. D, Scatter plot of individual voltage-clamp measurements of maximum summated uEPSC amplitudes in response to 5 uncaging pulses at $200 \mathrm{~Hz}$. E, Scatter plot of maximum summated uEPSP amplitudes in response to 5 uncaging pulses at $200 \mathrm{~Hz}$ as recorded optically from the spine and electrically from the soma. $\boldsymbol{F}$, Magnified display of uEPSP trains recorded with a somatic patch electrode (as in middle trace in D) indicating three typical examples of consistent linear summation of subthreshold signals at soma-axon region. 
$200 \mathrm{~Hz}$, mimicking the burst of APs in a presynaptic neuron. At the start of experiment, we made somatic electrical recordings of uEPSCs under voltage-clamp in response to repetitive activation of a spine synapse. Figure 4B illustrates a typical response showing that synaptic currents caused by individual uncaging pulses summated in a pronounced sublinear fashion. An equilibrium state was reached after the second pulse in this experiment and the maximum current during plateau phase reached a peak value of $70 \mathrm{pA}$. The summary data from a large series of similar measurements show that the average maximum summated current amplitude during the plateau phase was $52 \pm 1.7 \mathrm{pA}(\mathrm{n}=94)$. The distribution of individual values is shown in the scatter plot (Fig. 4D).

The voltage-clamp measurement of synaptic current was followed by optical recording of local uEPSPs in the current-clamp mode evoked by an identical uncaging protocol from the same spine on basal dendrite. Fig. 4C indicates a typical example of a prominent sublinear summation of local uEPSPs consistent with the summation profile of synaptic currents. The maximum response was reached after the second EPSP in this experiment, with the local depolarization reaching a plateau at $6 \mathrm{mV}$. At the same time, the summation of attenuated uEPSP signals measured at the soma with patch electrode was very nearly linear resulting in the maximum somatic depolarization of $3.5 \mathrm{mV}$. In a series of experiments of this kind, the average peak amplitude of the summated uEPSP at the site of origin was $8.8 \pm 1.0 \mathrm{mV}(\mathrm{n}=23)$. The corresponding summated uEPSP peak amplitude in the soma was $2.3 \pm 0.2 \mathrm{mV}(\mathrm{n}=56)$. The distribution of individual values of the summated uEPSP in the spine and in the soma is shown in the scatter plot in Fig. 4E. The RC filtering effect of basal dendrites promotes approximately linear summation at the soma-axon region by slowing the kinetic of EPSP signals as they propagate to the soma. Fig. $4 \mathrm{~F}$ shows three typical examples of linear summation of the train of 
uEPSPs as recorded in the soma with a patch electrode. An important advantage of this biophysical property of neurons is that it provides a mechanism for a wide dynamic range of near-linear integration of synaptic signals at the soma-axon region (the output end of the neuron) without saturating the driving force for synaptic current at remote local synapses on spines (the input end of the neuron).

Because individual synapses are the elementary computational units of the brain, it is important to understand biophysical properties that determine the sublinear temporal summation of subthreshold signals in dendritic spines. It is known that glutamate receptors on the postsynaptic side are far from saturation during quantal transmission (Liu et al., 1999; McAllister and Stevens, 2000). Thus, it is likely that AMPA receptor desensitization plays a major role in sublinear summation of unitary uEPSPs shown in Fig. 4. To test this prediction we monitored uEPSCs summation under voltage-clamp as recorded by a patch-electrode at the soma under control conditions and after AMPA receptor desensitization was inhibited by $100 \mu \mathrm{M}$ cyclothiazide (Partin et al., 1993) (Fig. 5). On average, addition of $100 \mu \mathrm{M}$ cyclothiazide increased the maximum synaptic current response to repetitive quantal release caused by 2photon uncaging of glutamate from $52 \pm 2.1 \mathrm{pA}$ to $177 \pm 21 \mathrm{pA}(\mathrm{n}=9)$, an increase in the mean value of $340 \%$. The scatter plot of the summary data (Fig. 5C) shows the distribution of individual values. The effect was clear and statistically significant as indicated by paired t-test (Fig. 5D). In cyclothiazide, with desensitization abolished, the synaptic current responses still summate in a sublinear mode, but the maximum current amplitude level is shifted toward larger values. In these conditions, the plateau is likely caused by receptor saturation. One would expect that, at saturation, almost all synaptic AMPA receptors are activated so that further application of glutamate will not increase synaptic current significantly. Indeed, this result is obtained when the 
train is extended from 5 to 10 uncaging pulses (Fig. 5E). The mean plateau value of the summated EPSC following 10 uncaging evoked releases of glutamate reached a value of $218 \pm 36$ pA, a small increase which was not statistically significant (t-test, $\mathrm{p}=0.48, \mathrm{n}=6)$ (Fig. 5F).

Following the somatic EPSC recordings in the voltage-clamp mode we determined the effects of desensitization block on the train of local EPSPs measured optically at the activated

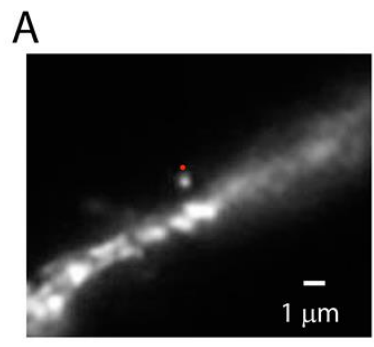

C

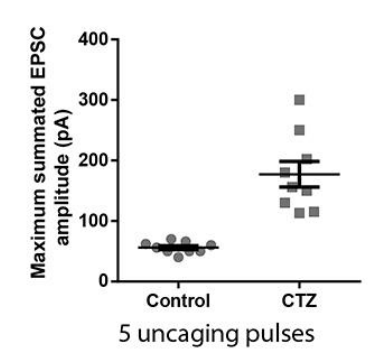

B
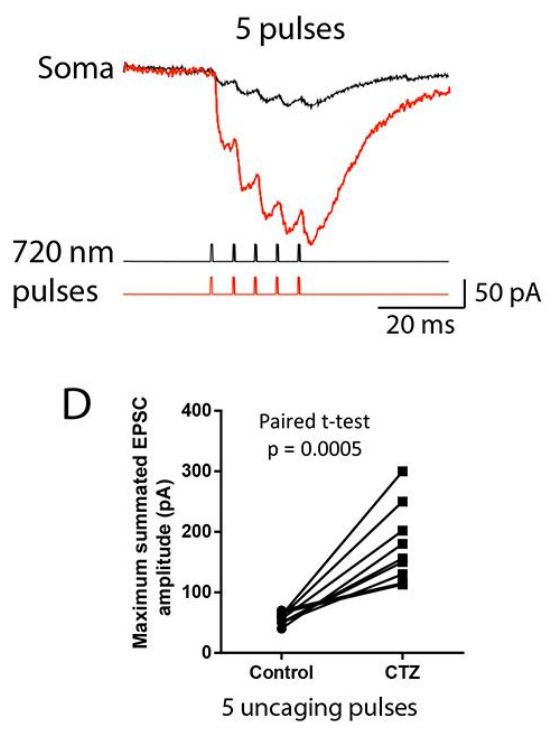

$\mathrm{E}$

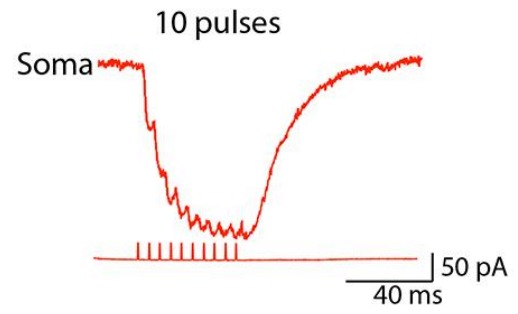

$\mathrm{F}$

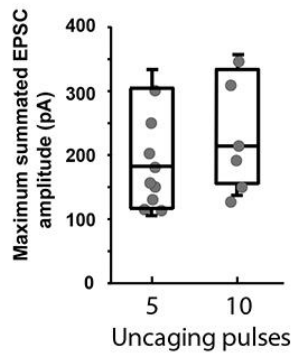

Figure 5. AMPAR desensitization underlies sublinear summation of unitary uEPSCs.

A, Fluorescence image of a spine in recording position. Red dot indicates the position the 720 $n m$ light spot used for glutamate uncaging. $\boldsymbol{B}$, Summating uEPSCs recorded with a somatic patch electrode under voltage-clamp in response to repetitive glutamate uncaging at $200 \mathrm{~Hz}$ under control conditions (black traces) and following bath application of AMPAR desensitization inhibitor cyclothiazide (red trace). Cyclothiazide caused a dramatic increased the maximum synaptic current response. Bottom traces: Uncaging command pulses. $\boldsymbol{C}$, Scatter plot showing distribution of data from individual experiments. $\boldsymbol{D}$, Paired $t$-test. $\boldsymbol{E}$, Summating uEPSCs recorded with a somatic patch electrode under voltage-clamp in response to 10 glutamate uncaging pulses at $200 \mathrm{~Hz}$ following bath application of cyclothiazide. $\boldsymbol{F}$, Comparison of mean plateau values of the summated EPSC following 5 and 10 uncaging pulses. 
dendritic spines as well as electrically with a somatic patch electrode (Fig. 6).
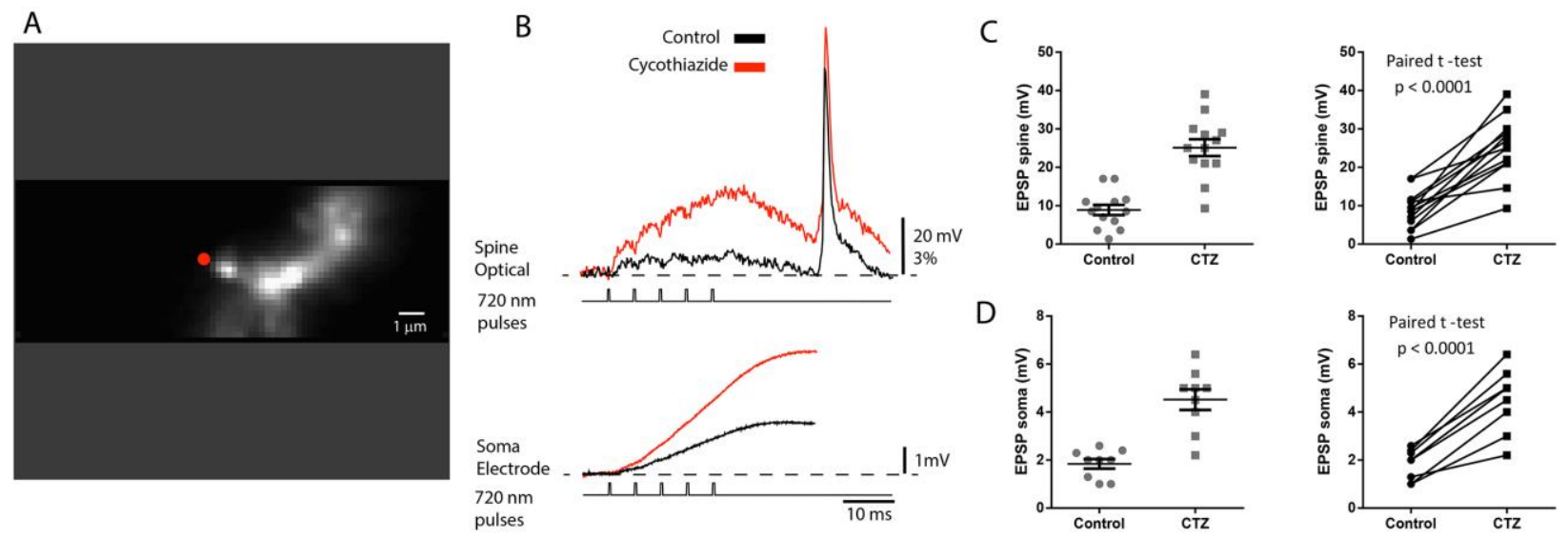

Figure 6. The effect of desensitization on summated train of uEPSPs. A, Fluorescence image of a spine in recording position. The red dot indicates the position of the $720 \mathrm{~nm}$ light spot used for 2-photon glutamate uncaging. $\boldsymbol{B}$, Upper traces: optical recordings of local summated uEPSP signals from the spine and parent dendrite under voltage-clamp evoked by repetitive glutamate uncaging at $200 \mathrm{~Hz}$. Black trace: control conditions. Red trace: bath application of $100 \mu \mathrm{M}$ AMPAR desensitization inhibitor cyclothiazide. Lower traces: simultaneous somatic patch electrode recordings. Bottom traces: uncaging command pulses. $\boldsymbol{C}$, Left panel: scatter plot of individual values of maximum summated uEPSP measured optically in the spine under control conditions and following bath application of cyclothiazide. Right panel: paired t-test shows significant difference. $\boldsymbol{D}$, Left panel: scatter plot of individual values of maximum summated uEPSP measured with somatic patch electrode under control conditions and following bath application of cyclothiazide. Vertical lines show mean \pm SEM. Right panel: paired t-test indicates significant difference.

The local EPSP response measured optically from dendritic spines and a small dendritic region at the base of the spines (Fig. 6) increased from $8.4 \pm 0.3 \mathrm{mV}$ in control solution to $25 \pm 3.4 \mathrm{mV}$ $(n=13)$, an increase of the mean value of $297 \%$, following bath application of cyclothiazide. The 
cyclothiazide increased summated EPSP response in the soma from $1.8 \pm 0.2 \mathrm{mV}$ to $4.4 \pm 0.4 \mathrm{mV}$ $(n=9)$, an increase of the average value of $244 \%$. The scatter plot of the summary data (Fig. $6 \mathrm{C}$ D) shows the distribution of individual data and high statistical significance of the effect.

\section{Discussion}

\section{EPSP transfer from spine to dendrite}

The presented results confirm and expand our prior findings (Popovic et al., 2015b) that the electrical resistance of the spine neck in L5 pyramidal neurons is too low, relative to the input impedance of the parent dendrite, to cause a voltage drop that would contribute significantly to the amplitude of the EPSP in the spine head. Similar experiments on L2/3 and L6 pyramidal neurons provided evidence that same conclusion is valid for these two additional classes of principal cortical neurons. These results rule out the electrical role of dendritic spines on examined pyramidal neurons. Our findings are based on measurements of the amplitude ratio of optical signals $A R=\mathrm{EPSP}_{\text {spine }} / \mathrm{EPSP}_{\text {dend }}=1+\left(\mathrm{R}_{\text {neck }} / \mathrm{Z}_{\text {dend }}\right)$ rather than on an attempt to measure absolute values of $\mathrm{R}_{\text {neck }}$ and $\mathrm{Z}_{\text {dend. }}$. The recorded values of the functional parameter $\mathrm{AR}$, which controls the synaptic weight, are very close to 1 for all three classes of cortical pyramidal neurons. This finding, derived directly from optical recordings with adequate sensitivity and spatiotemporal resolution, does not depend on any assumptions. It is also in agreement with theoretical predictions (Rall, 1974; Koch and Zador, 1993) as well as with diffusional resistance measurement which imply low $\mathrm{R}_{\text {neck }}$ relative to $\mathrm{Z}_{\mathrm{dend}}$ (Svoboda et al., 1996; Bloodgood and Sabatini, 2005; Takasaki and Sabatini, 2014; Tønnesen et al., 2014). A particularly strong indirect experimental evidence in agreement with our findings is the measurement of the 
diffusional resistance of the spine neck to $\mathrm{Na}^{+}$ion flux which mediates rapid removal of sodium from the spine head (Miyazaki and Ross, 2017). Due to the close analogy between diffusional coupling and the electrical resistance, it is difficult to argue against strong implications of diffusional measurements for the upper bound of the possible electrical resistance of the spine neck. An additional strong argument against the postulated high $\mathrm{R}_{\text {neck }}$ and electrical role of spines are two reports based on $\mathrm{Ca}^{2+}$ imaging that provided clear evidence for the lack of correlation between the morphology and dimensions of the spine neck and uncaging-evoked EPSPs and $\mathrm{Ca}^{2+}$ transients (Takasaki and Sabatini, 2014; Bywalez et al., 2015).

A fundamental implication of our findings is that spines on thin basal dendrites are characterized by uniform electrical behaviour regardless of considerable natural variations in their morphology under control conditions (Jones and Powell, 1969; Tønnesen et al., 2014). Thus, our data argue that relatively small changes in the morphology of individual single spines following induced synaptic plasticity (Takasaki et al., 2013; Araya et al., 2014; Tønnesen et al., 2014; Tazerart et al., 2020) are likely to be the by-product and not the cause of plastic changes. It follows that our results call into question several existing hypotheses on the electrical role of spines. For example, it has been postulated that spines may initiate APs (Araya et al., 2006b), that spines cause the reduction of location-dependent variability of local EPSP amplitude and normalize synaptic activation of NMDA receptors and voltage-gated channels (Gulledge et al., 2012), that spines amplify EPSPs up to 45-fold thus facilitating electrical interactions among coactive inputs and promoting associated forms of plasticity and storage (Harnett et al., 2012), that spine necks severely attenuate EPSP signals (Kwon et al., 2017), that spine geometry plays a key role in shaping the EPSP time course (Lagache et al., 2019), that spines are elementary 
voltage compartments that could be important for dendritic integration and disease states (Cornejo et al., 2022).

We consider out data to be the only direct evidence on electrical role of spines. In contrast, the experimental support for the above listed hypothesis is either circumstantial or based on measurements lacking sufficient accuracy. In our earlier paper (Popovic et al., 2015b) we discussed in detail the differences between our results and the interpretation of indirect evidence from a number of prior studies on spine neck resistance. This discussion will not be repeated here due to space constrains. In the meantime, several new studies interpreted their results to suggested that $R_{\text {neck }}$ is high relative to $Z_{\text {dend }}$ and that synapses on spine heads are electrically isolated from dendrites. In an optical recording study (Acker et al., 2016), the EPSP related signal could not be monitored in the spine head and in the dendrite simultaneously and the amplitude ratio across the spine neck (AR) could not be determined. Instead, the authors used a numerical simulation to approximate $\mathrm{R}_{\text {neck }}$ on an absolute scale (in $\mathrm{M} \Omega$ ). Given the uncertainties inherent to both modelling and estimates of $\mathrm{R}_{\text {neck }}$ from FRAP data based on cytosolic dyes, as well as the wide range of reported estimates of $\mathrm{R}_{\text {neck }}$ values (23-420 M $\Omega$ ), this study does not challenge our conclusions based on direct recordings. The first attempt at direct intracellular recordings from spines with nanopipette electrodes (Jayant et al., 2016) postulated that neck resistance is large enough to generate electrical compartmentalization of spines. This approach required extensive corrections and deconvolution procedures to reconstruct the true EPSP signals from recordings that were dramatically distorted by the high resistance of nanopipettes. Additionally, the electrical structure of miniscule spines is likely to be altered significantly by an attached electrode. Further improvements in this technology is needed before these results can be rigorously evaluated. Spine electrical properties were studied in cultured 
hippocampal neurons (Kwon et al., 2017) using a genetically encoded protein voltage sensor ArcLight (Jin et al., 2012). In contrast to our results, the main conclusion from these measurements was that EPSPs are severely attenuated as they propagate across the spine neck. The evidence in support of this conclusion is week because the accuracy of reported measurements suffers from several shortcomings. Firstly, the response time of the voltage indicator ArcLight is slow (10 msec in (Jin et al., 2012); $90 \mathrm{~ms}$ in (Bando et al., 2019). Consequently, the recorded EPSP signals in Kwon et al. (2017) have FWHH of 200 ms and bAP signals have FWHH of $~ 100 \mathrm{~ms}$. Clearly, an attempt to analyze electrical events that have a rise time of about $1 \mathrm{~ms}$ using a slow indicator and the acquisition frame rate of $100 \mathrm{~Hz}(10$ ms/data point) cannot generate accurate information. Furthermore, in the absence of an electrical signal to provide correct calibration of optical signals on an absolute scale (in $\mathrm{mV}$ ) this study relied on numerical simulation based on a kinetic model of voltage-dependent ArcLight fluorescence. The accuracy of calibration of profoundly distorted optical signals based on modeling is uncertain. Another recent effort to define the electrical role of spines in vivo (Cornejo et al., 2022) used two-photon microscopy and a genetically encoded protein voltage sensor to monitor electrical events in spines and dendrites during spontaneous activity and sensory stimulation. This approach is an impressive example of integrating several cutting-edge experimental techniques. However, because of poor temporal resolution and insufficent sensitivity of the voltage probe, the optical measurements could not distinguish either individual EPSPs or single APs from the noise. Thus, the conclusions were infered from heavily processed data that did not contain information on the characteristics of individual subthreshold or suprathreshold signals. It appears that further improvements in this technology will be needed before these results can be carefully evaluated. 


\section{Contribution of individual synapse to electrical signaling}

Using high-sensitivity voltage imaging, we obtained unique data on temporal summation of unitary EPSPs at the site of origin - i.e. at single excitatory synapses on dendritic spines. Our study shows that: (1) Temporal summation of repetitive quantal EPSP inputs at the site of origin (spine head) is markedly sublinear; (2) AMPAR desenzitization underlies the sublinear EPSP summation; (3) Distinctly sub-linear uEPSP summation at the synaptic site is paralleled by nearlinear summation at the soma-axon region; (4) Repetitive activation of individual synapses does not initiate APs at the soma-axon region or a dendritic spike, independently of input frequency.

In conclusion, local individual uEPSPs have fast kinetics resulting in little or no temporal summation at frequency below $100 \mathrm{~Hz}$. At higher frequencies $(200 \mathrm{~Hz})$, unitary EPSPs summate locally in a pronounced sub-linear manner. At the soma-axon region, EPSPs at both low and high frequency summate in near-linear fashion due to broadening of the EPSP signal caused by RC filtering in the dendrite. Thus, our study shows that AMPAR desensitization limits EPSP kinetics producing a sublinear temporal summation at the synaptic sites preventing depolarization buildup and synaptic saturation. This mechanism widens the dynamic range of near-linear summation of repetitive EPSPs at the soma-axon region.

\section{REFERENCES}

Acker CD, Hoyos E, Loew LM (2016) EPSPs Measured in Proximal Dendritic Spines of Cortical Pyramidal Neurons. Eneuro 3:1-13 Available at: http://eneuro.org/content/3/2/ENEURO.0050-15.2016.abstract.

Antić S, Zecević D (1995) Optical signals from neurons with internally applied voltage-sensitive dyes. J Neurosci 15:1392-1405 Available at: 
http://www.ncbi.nlm.nih.gov/pubmed/7869106 [Accessed July 29, 2014].

Araya R, Eisenthal KB, Yuste R (2006a) Dendritic spines linearize the summation of excitatory potentials. Proc Natl Acad Sci U S A 103:18799-18804 Available at:

http://www.pubmedcentral.nih.gov/articlerender.fcgi?artid=1693742\&tool=pmcentrez\&ren dertype $=$ abstract $[$ Accessed January 31, 2014].

Araya R, Jiang J, Eisenthal KB, Yuste R (2006b) The spine neck filters membrane potentials. Proc Natl Acad Sci U S A 103:17961-17966 Available at: http://www.pubmedcentral.nih.gov/articlerender.fcgi?artid=1693855\&tool=pmcentrez\&ren dertype $=$ abstract [Accessed January 22, 2014].

Araya R, Vogels TP, Yuste R (2014) Activity-dependent dendritic spine neck changes are correlated with synaptic strength. Proc Natl Acad Sci U S A 111:E2895-904 Available at: http://www.ncbi.nlm.nih.gov/pubmed/24982196 [Accessed July 9, 2014].

Bando Y, Sakamoto M, Kim S, Ayzenshtat I, Yuste R (2019) Comparative Evaluation of Genetically Encoded Voltage Indicators. Cell Rep 26:802-813.e4 Available at: https://doi.org/10.1016/j.celrep.2018.12.088.

Beaulieu-Laroche L, Harnett MT (2018) Dendritic Spines Prevent Synaptic Voltage Clamp. Neuron 97:75-82.e3.

Bloodgood BL, Giessel AJ, Sabatini BL (2009) Biphasic synaptic Ca influx arising from compartmentalized electrical signals in dendritic spines. PLoS Biol 7:e1000190 Available at:

http://www.pubmedcentral.nih.gov/articlerender.fcgi?artid=2734993\&tool=pmcentrez\&ren dertype=abstract [Accessed January 24, 2014]. 
Bloodgood BL, Sabatini BL (2005) Neuronal activity regulates diffusion across the neck of dendritic spines. Science 310:866-869 Available at:

http://www.ncbi.nlm.nih.gov/pubmed/16272125 [Accessed January 24, 2014].

Bywalez WG, Patirniche D, Rupprecht V, Stemmler M, Herz AVM, Pálfi D, Rózsa B, Egger V (2015) Local Postsynaptic Voltage-Gated Sodium Channel Activation in Dendritic Spines of Olfactory Bulb Granule Cells. Neuron 85:590-601 Available at: http://www.ncbi.nlm.nih.gov/pubmed/25619656 [Accessed January 27, 2015].

Cartailler J, Kwon T, Yuste R, Holcman D (2018) Deconvolution of Voltage Sensor Time Series and Electro-diffusion Modeling Reveal the Role of Spine Geometry in Controlling Synaptic Strength. Neuron 97:1126-1136.e10.

Carter AG, Sabatini BL (2004) State-dependent calcium signaling in dendritic spines of striatal medium spiny neurons. Neuron 44:483-493 Available at: http://www.ncbi.nlm.nih.gov/pubmed/15504328.

Chen X, Leischner U, Rochefort NL, Nelken I, Konnerth A (2011) Functional mapping of single spines in cortical neurons in vivo. Nature 475:501-505 Available at: http://dx.doi.org/10.1038/nature10193 [Accessed January 22, 2014].

Chiovini B, Turi GF, Katona G, Kaszás A, Pálfi D, Maák P, Szalay G, Szabó MF, Szabó G, Szadai Z, Káli S, Rózsa B (2014) Dendritic spikes induce ripples in parvalbumin interneurons during hippocampal sharp waves. Neuron 82:908-924 Available at: http://www.ncbi.nlm.nih.gov/pubmed/24853946 [Accessed July 11, 2014].

Cornejo VH, Ofer N, Yuste R (2022) Voltage compartmentalization in dendritic spines in vivo. Science (80- ) 375 . 
Enoki R, Hu Y-L, Hamilton D, Fine A (2009) Expression of long-term plasticity at individual synapses in hippocampus is graded, bidirectional, and mainly presynaptic: optical quantal analysis. Neuron 62:242-253 Available at: http://www.ncbi.nlm.nih.gov/pubmed/19409269 [Accessed October 27, 2014].

Grinvald A, Hildesheim R, Farber IC, Anglister L (1982) Improved fluorescent probes for the measurement of rapid changes in membrane potential. Biophys J 39:301-308 Available at: http://www.pubmedcentral.nih.gov/articlerender.fcgi?artid=1328947\&tool=pmcentrez\&ren dertype $=$ abstract [Accessed November 23, 2014].

Grunditz Å, Holbro N, Tian L, Zuo Y, Oertner TG, Grunditz A (2008) Spine Neck Plasticity Controls Postsynaptic Calcium Signals through Electrical Compartmentalization. J Neurosci 28:13457-13466 Available at: http://www.ncbi.nlm.nih.gov/pubmed/19074019 [Accessed January 23, 2014].

Gulledge AT, Carnevale NT, Stuart GJ (2012) Electrical advantages of dendritic spines. PLoS One 7:e36007 Available at: http://www.pubmedcentral.nih.gov/articlerender.fcgi?artid=3332048\&tool=pmcentrez\&ren dertype $=$ abstract $[$ Accessed January 31, 2014].

Gulyás AI, Megías M, Emri Z, Freund TF (1999) Total number and ratio of excitatory and inhibitory synapses converging onto single interneurons of different types in the CA1 area of the rat hippocampus. J Neurosci 19:10082-10097.

Hage TA, Sun Y, Khaliq ZM (2016) Electrical and Ca 2 + signaling in dendritic spines of substantia nigra dopaminergic neurons. Elife 5: e13905:1-26.

Harnett MT, Makara JK, Spruston N, Kath WL, Magee JC (2012) Synaptic amplification by dendritic spines enhances input cooperativity. Nature 491:599-602 Available at: 
http://dx.doi.org/10.1038/nature11554 [Accessed January 23, 2014].

Higley MJ, Sabatini BL, Long-term NR, Lüscher C, Malenka RC, Harris KM, Weinberg RJ, Smart TG, Paoletti P, Südhof C (2012) Calcium signaling in dendritic spines. Cold Spring Harb Perspect Biol 4:a005686 Available at:

http://www.pubmedcentral.nih.gov/articlerender.fcgi?artid=3312680\&tool=pmcentrez\&ren dertype=abstract [Accessed January 22, 2014].

Holthoff K, Zecevic D, Konnerth A (2010) Rapid time course of action potentials in spines and remote dendrites of mouse visual cortex neurons. J Physiol 588:1085-1096 Available at: http://www.pubmedcentral.nih.gov/articlerender.fcgi?artid=2852997\&tool=pmcentrez\&ren dertype $=$ abstract $[$ Accessed January 31, 2014].

Jayant K, Hirtz JJ, Plante IJ, Tsai DM, Boer WDAM De, Semonche A, Peterka DS, Owen JS, Sahin O, Shepard KL, Yuste R (2016) Targeted intracellular voltage recordings from dendritic spines using quantum-dot-coated nanopipettes. Nat Nanotechnol 12:335-342 Available at: http://dx.doi.org/10.1038/nnano.2016.268.

Jia H, Rochefort NL, Chen X, Konnerth A (2010) Dendritic organization of sensory input to cortical neurons in vivo. Nature 464:1307-1312 Available at: http://www.ncbi.nlm.nih.gov/pubmed/20428163 [Accessed January 20, 2014].

Jin L, Han Z, Platisa J, Wooltorton JRA, Cohen LB, Pieribone VA (2012) Single action potentials and subthreshold electrical events imaged in neurons with a fluorescent protein voltage probe. Neuron 75:779-785 Available at: http://www.pubmedcentral.nih.gov/articlerender.fcgi?artid=3439164\&tool=pmcentrez\&ren dertype $=$ abstract [Accessed July 18, 2014]. 
Jones EG, Powell TP (1969) Morphological variations in the dendritic spines of the neocortex. J

Cell Sci 5:509-529 Available at: http://www.ncbi.nlm.nih.gov/pubmed/5362339.

Koch C, Poggio T, B PRSL (1983) A Theoretical Analysis of Electrical Properties of Spines.

Proc R Soc B Biol Sci 218:455-477 Available at:

http://rspb.royalsocietypublishing.org/cgi/doi/10.1098/rspb.1983.0051 [Accessed January

$31,2014]$.

Koch C, Zador A (1993) The function of dendritic spines: devices subserving biochemical rather than electrical compartmentalization. J Neurosci 13:413-422 Available at:

http://www.ncbi.nlm.nih.gov/pubmed/8426220 [Accessed August 23, 2014].

Kole MHP (2011) First node of Ranvier facilitates high-frequency burst encoding. Neuron

71:671-682 Available at: http://dx.doi.org/10.1016/j.neuron.2011.06.024 [Accessed January $31,2014]$.

Kuhn B, Fromherz P, Denk W (2004) High sensitivity of Stark-shift voltage-sensing dyes by one- or two-photon excitation near the red spectral edge. Biophys J 87:631-639 Available at:

http://www.pubmedcentral.nih.gov/articlerender.fcgi?artid=1304385\&tool=pmcentrez\&ren dertype $=$ abstract $[$ Accessed January 31, 2014].

Kwon T, Sakamoto M, Peterka DS, Yuste R (2017) Attenuation of Synaptic Potentials in Dendritic Spines. Cell Rep 20:1100-1110 Available at: http://dx.doi.org/10.1016/j.celrep.2017.07.012.

Lagache T, Jayant K, Yuste R (2019) Electrodiffusion models of synaptic potentials in dendritic spines. J Comput Neurosci 47:77-89.

Liu G, Choi S, Tsien RW (1999) Variability of neurotransmitter concentration and nonsaturation 
of postsynaptic AMPA receptors at synapses in hippocampal cultures and slices. Neuron 22:395-409 Available at: http://www.ncbi.nlm.nih.gov/pubmed/10069344.

Loew LM (1982) Design and characterization of electrochromic membrane probes. J Biochem Biophys Methods 6:243-260 Available at: http://www.ncbi.nlm.nih.gov/pubmed/7130621.

Magee JC, Cook EP (2000) Somatic EPSP amplitude is independent of synapse location in hippocampal pyramidal neurons. Nat Neurosci 3:895-903 Available at: http://www.ncbi.nlm.nih.gov/pubmed/10966620.

Matsuzaki M, Ellis-davies GCR, Nemoto T, Miyashita Y, Iino M, Kasai H (2001) Dendritic spine geometry is critical for AMPA receptor expression in hippocampal CA1 pyramidal neurons. Nat Neurosci 4:1086-1092 Available at: http://www.ncbi.nlm.nih.gov/pubmed/11687814 [Accessed January 23, 2014].

McAllister a K, Stevens CF (2000) Nonsaturation of AMPA and NMDA receptors at hippocampal synapses. Proc Natl Acad Sci U S A 97:6173-6178 Available at: http://www.pubmedcentral.nih.gov/articlerender.fcgi?artid=18577\&tool=pmcentrez\&render type $=$ abstract

Miyazaki K, Ross WN (2017) Sodium Dynamics in Pyramidal Neuron Dendritic Spines: Synaptically Evoked Entry Predominantly through AMPA Receptors and Removal by Diffusion. J Neurosci 37:9964-9976 Available at: http://www.jneurosci.org/lookup/doi/10.1523/JNEUROSCI.1758-17.2017.

Nevian T, Larkum ME, Polsky A, Schiller J (2007) Properties of basal dendrites of layer 5 pyramidal neurons: a direct patch-clamp recording study. Nat Neurosci 10:206-214 Available at: http://www.ncbi.nlm.nih.gov/pubmed/17206140 [Accessed January 31, 2014]. 
Palmer LM, Stuart GJ (2009) Membrane potential changes in dendritic spines during action potentials and synaptic input. J Neurosci 29:6897-6903 Available at: http://www.ncbi.nlm.nih.gov/pubmed/19474316 [Accessed January 31, 2014].

Partin KM, Patneau DK, Winters C a, Mayer ML, Buonanno a (1993) Selective modulation of desensitization at AMPA versus kainate receptors by cyclothiazide and concanavalin A. Neuron 11:1069-1082 Available at: http://www.ncbi.nlm.nih.gov/pubmed/7506043.

Popovic M, Vogt K, Holthoff K, Konnerth A, Salzberg BM, Grinvald A, Antic SD, Canepari M, Zecevic D (2015a) Imaging submillisecond membrane potential changes from individual regions of single axons, dendrites and spines. In: Membrane Potential Imaging in the Nervous System and Heart, pp 57-101.

Popovic MA, Carnevale N, Rozsa B, Zecevic D (2015b) Electrical behaviour of dendritic spines as revealed by voltage imaging. Nat Commun 6:8436 Available at: http://www.nature.com/ncomms/2015/151005/ncomms9436/full/ncomms9436.html?WT.ec _id=NCOMMS-

20151007\&spMailingID=49717544\&spUserID=NDI1Mzg0NDg0NgS2\&spJobID=780943 740\&spReportId=NzgwOTQzNzQwS0.

Popovic MA, Foust AJ, Mccormick D a, Zecevic D (2011) The spatio-temporal characteristics of action potential initiation in layer 5 pyramidal neurons: a voltage imaging study. J Physiol 589:4167-4187 Available at: http://www.pubmedcentral.nih.gov/articlerender.fcgi?artid=3180577\&tool=pmcentrez\&ren dertype $=$ abstract $[$ Accessed January 31, 2014].

Popovic MA, Gao X, Carnevale NT, Zecevic D (2014) Cortical dendritic spine heads are not electrically isolated by the spine neck from membrane potential signals in parent dendrites. 
Cereb Cortex 24:385-395 Available at: http://www.ncbi.nlm.nih.gov/pubmed/23054810

[Accessed January 31, 2014].

Rall W (1974) Cellular mechanisms subserving changes in neuronal activity (Segev, I, Rinzel, J, Shepherd G, ed). Cambridge, MA: MIT press.

Rall W, Rinzel J (1973) Branch input resistance and steady attenuation for input to one branch of a dendritic neuron model. Biophys J 13:648-687 Available at: http://www.pubmedcentral.nih.gov/articlerender.fcgi?artid=1484314\&tool=pmcentrez\&ren dertype=abstract [Accessed January 31, 2014].

Sancho L, Bloodgood BL (2018) Functional Distinctions between Spine and Dendritic Synapses Made onto Parvalbumin-Positive Interneurons in Mouse Cortex. Cell Rep 24:2075-2087 Available at: https://doi.org/10.1016/j.celrep.2018.07.070.

Smith M a, Ellis-Davies GCR, Magee JC (2003) Mechanism of the distance-dependent scaling of Schaffer collateral synapses in rat CA1 pyramidal neurons. J Physiol 548:245-258 Available at: http://www.pubmedcentral.nih.gov/articlerender.fcgi?artid=2342790\&tool=pmcentrez\&ren dertype $=$ abstract [Accessed January 31, 2014].

Sobczyk A, Scheuss V, Svoboda K (2005) NMDA receptor subunit-dependent [Ca2+] signaling in individual hippocampal dendritic spines. J Neurosci 25:6037-6046 Available at: http://www.ncbi.nlm.nih.gov/pubmed/15987933 [Accessed January 31, 2014].

Stuart G, Spruston N (1998) Determinants of voltage attenuation in neocortical pyramidal neuron dendrites. J Neurosci 18:3501-3510 Available at: http://www.ncbi.nlm.nih.gov/pubmed/9570781. 
Svoboda K, Tank DW, Denk W (1996) Direct measurement of coupling between dendritic spines and shafts. Science 272:716-719 Available at: http://www.ncbi.nlm.nih.gov/pubmed/8614831 [Accessed August 24, 2014].

Takasaki K, Sabatini BL (2014) Super-resolution 2-photon microscopy reveals that the morphology of each dendritic spine correlates with diffusive but not synaptic properties. Front Neuroanat 8:29 Available at: http://www.pubmedcentral.nih.gov/articlerender.fcgi?artid=4019874\&tool=pmcentrez\&ren dertype $=$ abstract [Accessed July 10, 2014].

Takasaki KT, Ding JB, Sabatini BL (2013) Live-cell superresolution imaging by pulsed STED two-photon excitation microscopy. Biophys J 104:770-777 Available at: http://dx.doi.org/10.1016/j.bpj.2012.12.053 [Accessed January 27, 2014].

Tazerart S, Mitchell DE, Miranda-Rottmann S, Araya R (2020) A spike-timing-dependent plasticity rule for dendritic spines. Nat Commun 11 Available at: http://dx.doi.org/10.1038/s41467-020-17861-7.

Tønnesen J, Katona G, Rózsa B, Nägerl UV (2014) Spine neck plasticity regulates compartmentalization of synapses. Nat Neurosci 17:678-685 Available at: http://www.ncbi.nlm.nih.gov/pubmed/24657968 [Accessed July 10, 2014].

Varga Z, Jia H, Sakmann B, Konnerth A (2011) Dendritic coding of multiple sensory inputs in single cortical neurons in vivo. Proc Natl Acad Sci U S A 108:15420-15425 Available at: http://www.pubmedcentral.nih.gov/articlerender.fcgi?artid=3174623\&tool=pmcentrez\&ren dertype $=$ abstract [Accessed January 25, 2014].

Xu N, Harnett MT, Williams SR, Huber D, O’Connor DH, Svoboda K, Magee JC, Connor DHO (2012) Nonlinear dendritic integration of sensory and motor input during an active sensing 
task. Nature 492:247-251 Available at: http://dx.doi.org/10.1038/nature11601 [Accessed

January 23, 2014].

Zecević D (1996) Multiple spike-initiation zones in single neurons revealed by voltage-sensitive

dyes. Nature 381:322-325 Available at: http://www.ncbi.nlm.nih.gov/pubmed/8692270

[Accessed July 29, 2014]. 